05,11

\title{
Магнитные и магнитокалорические эффекты в системах с реверсивными переходами первого рода
}

\author{
() В.И. Вальков ${ }^{1}$, В.И. Каменев 1 , А.В. Головчан ${ }^{1}$, И.Ф. Грибанов ${ }^{1}$, В.В. Коледов ${ }^{2}$, \\ В.Г. Шавров ${ }^{2}$ В.И. Митюк ${ }^{3}$, П. Дуда ${ }^{4}$ \\ ${ }^{1}$ Донецкий фризико-технический институт им. А.А. Галкина, \\ Донецк, Украина \\ ${ }^{2}$ Институт радиотехники и электроники им. В.А. Котельникова РАН, \\ Москва, Россия \\ ${ }^{3}$ НПЦ НАН Беларуси по материаловедению, \\ Минск, Беларусь \\ ${ }^{4}$ Варшавский политехнический университет, \\ 00-662 Варшава, Польша \\ E-mail: valkov09@gmail.com
}

Поступила в Редакцию 25 декабря 2020 г.

В окончательной редакции 25 декабря 2020 г.

Принята к публикации 26 декабря 2020 г.

В рамках модели взаимодействующих параметров магнитного и структурного порядков проведен теоретический анализ реверсивных магнитоструктурных фазовых переходов первого рода. Характерной особенностью последних является скачкообразное возникновение магнитного порядка при охлаждении, как в случае фазового перехода первого рода, и плавное исчезновение магнитного порядка при нагреве, как и в традиционном фазовом переходе второго рода. Такие переходы наблюдаются в некоторых сплавах магнитокалорической системы $\mathrm{Mn}_{1-x} \mathrm{Cr}_{x} \mathrm{NiGe}$ под давлением $(x=0.11)$ и без него $(x=0.18)$ и сопровождаются специфическими магнитными и магнитокалорическими особенностями. Феноменологическое описание этих особенностей проводится в рамках концепции мягкой моды для структурной подсистемы, претерпевающей структурный фазовый переход первого рода $\left(P 6_{3} / m m c-P n m a\right)$, и модели Гейзенберга для спиновой подсистемы. Для систем с магнитоструктурной неустойчивостью в рамках приближения молекулярного поля для спиновой подсистемы и приближения смещенного гармонического осциллятора для решеточной подсистемы показано, что реверсивные фазовые переходы возникают, когда температура магнитного разупорядочения находится в области температурного гистерезиса структурного фазового перехода первого рода $P 6_{3} / m m c-P n m a$. Также показано, что двухпиковая форма изотермической энтропии, характерная для реверсивных переходов, обусловлена разделением вкладов структурной и магнитной энтропии.

Ключевые слова: магнитоструктурный переход, гелимагнетизм, реверсивные переходы первого рода.

DOI: 10.21883/FTT.2021.05.50813.271

\section{1. Введение}

Фазовые переходы в системах с магнитной и кристаллоструктурной неустойчивостью при определенных условиях могут протекать как реверсивные магнитоструктурные переходы первого рода. Для таких переходов при понижении температуры возникновение магнитного порядка реализуется скачком, характерным для переходов первого рода, а при обратном повышении температуры плавное исчезновение магнитного порядка происходит как традиционный переход второго рода. Подобные переходы сопровождаются рядом магнитных и магнитокалорических особенностей. Настоящая работа является теоретическим анализом цикла экспериментальных исследований барических и магнитокалорических особенностей, характерных для реверсивных переходов. Для спиновой подсистемы в работе дано более подробное обоснование подхода, развитого в нашей предыдущей публикации в ФТТ, посвященной магнитоструктурным особенностям системы $\mathrm{Mn}_{1-x} \mathrm{Cr}_{x} \mathrm{NiGe}$.

\section{2. Экспериментальные результаты}

Как следует из результатов экспериментальных исследований, особенности магнитных и магнитокалорических свойств сплавов системы $\mathrm{Mn}_{1-x} \mathrm{Cr}_{x} \mathrm{NiGe}$ определяются относительной близостью температур $T_{t 2}(x) \quad$ и $T_{t 1}(x)$ структурного парамагнитного (PM) перехода первого рода $P M\left(P 6_{3} / m m c\right) \leftrightarrow P M(P n m a)$ и температур $T_{\mathrm{N}}(x), \quad T_{\mathrm{C}}(x)$ магнитных фазовых переходов парамагнетизм-гелимагнетизм $\mathrm{HM})$ $P M($ Pnma $) \leftrightarrow H M($ Pnma $), \quad$ парамагнетизм-ферромагнетизм (FM) $P M($ Pnma $) \leftrightarrow F M($ Pnma $) \quad[1,2]$. При этом структурные переходы могут фиксироваться по аномальному изменению магнитных характеристик в 
окрестности температур структурного перехода $T_{t 2}(x)$, $T_{t 1}(x)$, например, намагниченности $\sigma$ и обратной магнитной восприимчивости $\chi^{-1}[3]$. Отчетливая корреляция между высокотемпературными аномалиями зависимостей $\chi^{-1}(T), \sigma(T)$ и температурной зависимостью содержания орторомбической фазы в образце $X$-int $(T)$, обнаруженная в [1-3], позволили зафиксировать в магнитном поле конечной напряженности скачкообразное поведение высокотемпературного участка намагниченности. Эти скачкообразные изменения намагниченности, сопровождающиеся температурным гистерезисом, являются периферийными магнитоструктурными фазовыми переходами первого рода и, согласно результатам работы [3], обусловлены смещением температуры $T_{t 2}(x)$ структурного перехода $\operatorname{orth}\left(P_{n m a}\right) \rightarrow h e x\left(P 6_{3} / m m c\right)$ под действием магнитного поля.

На основании результатов [3] и данных [4] можно констатировать, что сближение структурного и магнитного переходов, возникающее при увеличении $x$, приводит к изменению типа магнитоупорядоченных фаз и характера магнитных фазовых переходов от изоструктурного безгистерезисного перехода второго рода $P M($ orth $) \leftrightarrow H M($ orth $)$ в окрестности $T_{\mathrm{N}} \geq \theta_{\text {orth }}$ из подмагниченного парамагнитного ромбического состояния в подмагниченное ромбическое геликоидальное состояние при $x=0.11$ к реверсивному магнитоструктурному переходу первого рода $P M($ hex $) \leftrightarrow F M($ orth $)$ при $x=0.18$. Реверсивный переход является суперпозицией двух фазовых переходов. При понижении температуры до $T_{\mathrm{C} 1}=T_{t 1}<\theta_{\text {orth }}$ происходит магнитоструктурный переход первого рода $P M($ hex $) \rightarrow F M($ orth $)$. Последующее нагревание до $T_{\mathrm{C} 2}=\theta_{\text {orth }}<T_{t 2}$ приводит к плавному магнитному разупорядочению с сохранением ромбической фазы. Это изоструктурное разупорядочение может интерпретироваться как изоструктурный магнитный фазовый переход второго рода $F M($ orth $) \rightarrow P M($ orth $)$.

\section{3. Теория реверсивных переходов в системе $\mathrm{Mn}_{1-x} \mathrm{Cr}_{x} \mathrm{NiGe}$}

Теоретический анализ структурных переходов типа „смещения“ опирается на результаты работы [3], в которой парамагнитные структурные переходы $P M\left(P 6_{3} / m m c\right) \leftrightarrow P M(P n т а)$ рассматриваются в рамках теории мягкой моды как следствие замораживания коррелированных локальных оптических колебаний ионов Ni. Термодинамика спиновой подсистемы и ее взаимосвязь со структурными параметрами порядка описывалась в [3] гамильтонианом Гейзенберга, что позволило описать наблюдаемое расщепление $\chi^{-1}(T)$ для случая, когда исходные гексагональное и ромбическое магнитоупорядоченные состояния являются геликоидальными и предсказать изменение характера магнитных фазовых переходов из парамагнитного в магнитоупорядоченное состояние при наложении гидростатического давления.
Согласно модели [3], гамильтониан можно представить в виде

$$
\hat{\mathbf{H}}=\mathbf{H}\left(Q_{n}\right)+\mathbf{H}\left(e_{1}, e_{2}\right)+\hat{\mathbf{H}}(s) .
$$

Тут $\mathbf{H}\left(Q_{n}\right)$ - эффективный гамильтониан, который описывает кооперативные локальные смещения атомов $\mathrm{Ni}$ в $n$-й гексагональной ячейке $Q_{n}$ в результате структурного перехода $P M\left(P 6_{3} / m m c\right) \leftrightarrow P M\left(P_{n m a}\right)$. Гамильтониан $\mathbf{H}\left(Q_{n}\right)$ включает гармонические $\left(V_{0} Q_{n}^{2}\right)$ и ангармонические $\left(\gamma Q_{n}^{4} / 4+\Gamma Q_{n}^{6} / 6\right)$ слагаемые потенциальной энергии внутри ячейки и гармонические взаимодействия между смещениями различных гексагональных ячеек $v_{n n^{\prime}} Q_{n} Q_{n^{\prime}}$, где $V_{0}$ и $v_{n, n^{\prime}}$ - энергии гармонических смещений атомов $\mathrm{Ni}$ в ячейке и между ячейками, $\gamma, \Gamma-$ энергии ангармонических смещений атомов $\mathrm{Ni}$ внутри элементарной ячейки.

Упругие деформации $e_{1}$ и $e_{2}$, которые описывают относительное изменение объема при изменении температуры $T$ и давления $P$ и ромбические искажения гексагональной ячейки [3], учитываются слагаемым $\mathbf{H}\left(e_{1}, e_{2}\right)$.

Магнитная структура и кооперативные явления в спиновой подсистеме описываются в рамках „гейзенберговского“ подхода оператором $\hat{\mathbf{H}}(s)$ в предположении, что эффективные обменные интегралы ${ }_{n n^{\prime}}^{i i^{\prime}}$ между соседними квазилокализованными спинами могут быть определены из первопринципных расчетов через зависимость полной энергии магнетика от ориентации магнитных моментов [7-9].

Магнитные моменты в $\mathrm{Mn}_{1-x} \mathrm{Cr}_{x} \mathrm{NiGe}$ создаются коллективизированными электронами, и возникновение ромбической структуры, приводящее к удвоению периода элементарной ячейки, проявляется прежде всего в расщеплении плотности состояний $d$-электронов. Это обеспечивает взаимосвязь между магнитной и структурной подсистемами. При использовании гамильтониана Гейзенберга для описания такой системы вышеуказанная взаимосвязь возникает при введении зависимости величины интегралов обменного взаимодействия от конфигурации атомов кристаллической решетки, которая определяется гексагональной hex $\left(P 6_{3} / m m c\right)$ или ромбической $\operatorname{orth}\left(P_{n m a}\right)$ симметрией кристаллической структуры.

Используя приближение среднего поля для спинового гамильтониана и приближение смещенного гармонического осциллятора $[3,6]$ для гамильтониана со взаимодействующими мягкими модами, термодинамический потенциал (ТП) магнитоупругой системы можно представить в виде суммы

$$
\begin{aligned}
& \Omega\left(Q_{0}, y, e_{1}, e_{2}\right)=\Omega_{1}\left(Q_{0}, \sigma, e_{1}, e_{2}\right)+\Omega_{2}(y)+\Omega_{3}\left(e_{1}, e_{2}\right), \\
& \Omega_{1}\left(Q_{0}, \sigma, e_{1}, e_{2}\right)=\frac{N_{0}}{2} V_{0}\left(Q_{0}^{2}+\sigma\right)+\frac{N_{0}}{4}\left(\gamma Q_{0}^{4}+6 Q_{0}^{2} \sigma+3 \sigma^{2}\right) \\
& +\frac{N_{0}}{6} \Gamma\left(Q_{0}^{6}+15 Q_{0}^{4} \sigma+45 Q_{0}^{2} \sigma^{2}+15 \sigma^{3}\right) \\
& -\frac{1}{2} N_{0} Q_{0}^{2} v_{0}\left(1+L_{1} e_{1}+L_{2} e_{2}\right)-T \frac{k_{B}}{2} N_{0} \ln \sigma,
\end{aligned}
$$




$$
\Omega_{3}\left(e_{1}, e_{2}\right)=\frac{1}{2} e_{1}^{2} k_{0}+\frac{1}{2} k_{1}\left(e_{2}\right)^{2}+P e_{1}-T \alpha k_{0} e_{1},
$$

$$
\Omega_{2}(y)=N s^{2}\left[J(\mathbf{k}) \sin ^{2} \vartheta+J(0) \cos ^{2} \vartheta\right] y^{2}-N k_{B} T \ln z(X),
$$

$$
z(X)=\operatorname{sh}\left[\left(1+(2 s)^{-1}\right) X\right] / \operatorname{sh}\left[(2 s)^{-1} X\right], \mathbf{k}=\left(0,0, k_{a}\right) .
$$

$$
\begin{aligned}
X= & {\left[2 s^{2} y\left[J\left(k_{\mathrm{a}}\right) \sin ^{2}(\vartheta)+J(0) \cos ^{2}(\vartheta)\right]\right.} \\
& \left.+2 \mu_{0} s H_{0} \cos \vartheta\right] / k_{B} T
\end{aligned}
$$

$$
\begin{aligned}
J(\mathbf{k}) & =J\left(k_{a}\right) \\
& =J_{0}\left(Q_{0}, e_{1}\right)+J_{1}\left(Q_{0}, e_{1}\right) \cos \Psi+J_{2}\left(Q_{0}, e_{1}\right) \cos 2 \Psi,
\end{aligned}
$$

$$
J(0)=J_{0}\left(Q_{0}, e_{1}\right)+J_{1}\left(Q_{0}, e_{1}\right)+J_{2}\left(Q_{0}, e_{1}\right)
$$

Здесь $\mathbf{k}=\left(0,0, k_{a}\right)$-волновой вектор геликоидальной структуры; $k_{0}, k_{1}$ и $\alpha-$ соответствующие упругие модули и коэффициент объемного температурного расширения; $v_{0}=\sum_{n} v_{n n^{\prime}} ; \quad L_{1}=\left(\partial v_{0} / \partial e_{1}\right) / v_{0}$, $L_{2}=\left(\partial v_{0} / \partial e_{2}\right) / v_{0}, \quad k_{B}-{ }^{n}$ постоянная Больцмана, $\sum_{n}=N_{0}, \sum_{n, k}=N=2 N_{0}(1-x)-$ число элементарных ячеек и число магнитоактивных атомов $(\mathrm{Mn})$ в единице объема. В приложении показан вывод ТП спиновой подсистемы $\Omega(y)$.

Среднее значение $Q_{0}=\int_{-\infty}^{\infty} \frac{1}{\sqrt{2 \pi \sigma}} \exp \left[\frac{-\left(Q_{n}-Q_{0}\right)^{2}}{2 \sigma}\right] Q_{n} d Q_{n}$, отождествляемое с параметром структурного порядка, и дисперсия $\sigma=\left\langle\left[Q_{n}-Q_{0}\right]^{2}\right\rangle$ рассматриваются как независимые вариационные параметры и определяются из минимизации термодинамического потенциала магнитоупругой системы $\Omega\left(Q_{0}, \sigma, e_{1}, e_{2}, y\right) ; s-$ собственное значение оператора спина, $y=\left|\left\langle\hat{\mathbf{s}}_{n}^{i}\right\rangle\right| / s$ - относительное значение магнитного момента $i$-го атома $\mathrm{Mn}$, соответствующее параметру магнитного порядка в геликоидальном состоянии; $\vartheta-$ угол между направлением локальной оси квантования и внешним магнитным полем $\mathbf{H}_{0}$; $\Psi=k_{a} \pi$ и $2 \Psi-$ углы между направлением средних значений спинов атомов $\left\langle\hat{\mathbf{s}}_{n}^{k}\right\rangle$, которые расположены на расстояниях $c_{h} / 2$ и $c_{h}\left(c_{h}-\right.$ параметр гексагональной ячейки вдоль направления волнового вектора $\mathbf{k}$ ); $J_{0}\left(Q_{0}, e_{1}\right), J_{1}\left(Q_{0}, e_{1}\right), J_{2}\left(Q_{0}, e_{1}\right)$ - межатомные обменные интегралы внутри ферромагнитного слоя и между ближайшими слоями на расстояниях $c_{h} / 2$ и $c_{h}$.

При $J_{1}\left(Q_{0}, e_{1}\right)>0, J_{2}\left(Q_{0}, e_{1}\right)<0$ конкурирующими состояниями будут только геликоидальное $\left(\cos \Psi=J_{1}\left(Q_{0}, e_{1}\right) / 4\left|J_{2}\left(Q_{0}, e_{1}\right)\right|=\delta<1\right) \quad$ с $\quad$ более высоким значением величины $J(k)\left(J(k)=J_{0}\left(Q_{0}, e_{1}\right)\right.$ $\left.+\left(2 \delta^{2}+1\right)\left|J_{2}\left(Q_{0}, e_{1}\right)\right|\right)$ и ферромагнитное $(\Psi=0)$ c более низким значением величины $J(0)$. Величины $J(k)$, $J(0)$ можно представить в виде разложения по линейным комбинациям деформаций и четным степеням парамет- ров структурного порядка:

$$
\begin{aligned}
J\left(k_{a}\right) & =J_{00}\left(1+\lambda_{0 e} e_{1}+Q_{0}^{2}\left(\lambda_{0 Q}+\lambda_{0 e Q} e_{1}\right)+\lambda_{4} Q_{0}^{4}\right) \\
& +\left|J_{20}\right|\left(1+Q_{0}^{2}\left(\lambda_{2 Q}+\lambda_{2 e Q} e_{1}\right)+\lambda_{2 e} e_{1}\right)\left(2 \delta^{2}+1\right), \\
J(0) & =J_{00}\left(1+\lambda_{0 e} e_{1}+Q_{0}^{2}\left(\lambda_{0 Q}+\lambda_{0 e Q} e_{1}\right)+\lambda_{4} Q_{0}^{4}\right) \\
& +\left|J_{20}\right|\left(1+Q_{0}^{2}\left(\lambda_{2 Q}+\lambda_{2 e Q} e_{1}\right)+\lambda_{2 e} e_{1}\right)(4 \delta-1),
\end{aligned}
$$

В (5) $J_{00}, J_{20}-$ соответствующие межатомные обменные интегралы в недеформированной $\left(e_{1}=0\right)$ гексагональной $\left(Q_{0}=0\right)$ системе. Величина

$$
\begin{aligned}
\delta=\cos \Psi= & J_{10}\left(1+Q_{0}^{2}\left(\lambda_{1 Q}+\lambda_{1 e Q} e_{1}\right)+\lambda_{1 e} e_{1}\right) / 4\left|J_{20}\right| \\
& \times\left(1+Q_{0}^{2}\left(\lambda_{2 Q}+\lambda_{2 e} e_{1}\right)+\lambda_{2 e} e_{1}\right)
\end{aligned}
$$

приближенно определяется как постоянная $\left(\delta=J_{10} / 4\left|J_{20}\right|\right)$. Согласно данным нейтронографических исследований [4], такое допущение приемлемо для исследуемых в настоящей работе образцов с $x=0.18$ $(\delta \approx 1)$ и $x=0.11 \quad(\delta \approx 0.87-0.93)$. Температурные зависимости магнитных, структурных и упругих характеристик можно получить из уравнений экстремума термодинамического потенциала: $\partial \Omega / \partial \vartheta=0, \partial \Omega / \partial y=0$, $\partial \Omega / \partial Q_{0}=0, \partial \Omega / \partial \sigma=0, \partial \Omega / \partial e_{1}=0, \partial \Omega / \partial e_{2}=0$.

Численное решение уравнений $\partial \Omega / \partial Q_{0}=0$, $\partial \Omega / \partial y=0$ наряду $\quad$ c аналитическими решениями остальных уравнений показывают, что при достаточно сильном взаимодействии между фононной подсистемой и упругими деформациями $\left(L_{1} \geq L_{1 k}>0, L_{2} \geq L_{2 k}\right)$ Зависимость $Q_{0}(T)$ будет описывать переход первого рода; для температур лабильности при этом, согласно экспериментальным данным, должны выполняться неравенства $\partial T_{t 1,2} / \partial P<0, \quad \partial\left(T_{t 2}-T_{t 1}\right) / \partial P<0 . \quad$ Если к тому же обеспечить выполнение неравенств $T_{t 1}>T_{\text {North }} \geq \theta_{\text {orth }} \ldots \quad$ (здесь $T_{\text {North }} \quad$ и $\quad \theta_{\text {orth }}-$ температура Нееля и парамагнитная температура Кюри в ромбической фазе) путем подгонки наборов коэффициентов $\lambda_{j e}, \lambda_{j Q}, \lambda_{j e Q}(j=0,1,2)$, то можно перейти к анализу нетривиальных особенностей поведения магнитоструктурных характеристик в исследуемой системе. Отметим, что в дальнейшем мы полагаем $\lambda_{0 e}=\lambda_{2 e}=0$. Тогда для безразмерной восприимчивости $\chi^{-1}\left(T, Q_{0}\right)=2 \mu_{0}(s+1) H_{0} / k_{B} T_{0} y \cos \vartheta$ при $H_{0} \rightarrow 0$ из уравнения $\partial \Omega / \partial y=0$ можно получить выражение

$$
\begin{aligned}
& \chi^{-1}\left(T, Q_{0}\right)=\frac{T}{T_{0}}-\left[r F(\delta)+Q_{0}^{2}\right. \\
&\left.\times\left[\lambda_{F}+\left(\alpha T-P \kappa+\frac{\nu_{0} L_{1} Q_{0}^{2} \kappa}{2}\right) \lambda_{1 F}\right]+\lambda_{4} Q_{0}^{4}\right],
\end{aligned}
$$



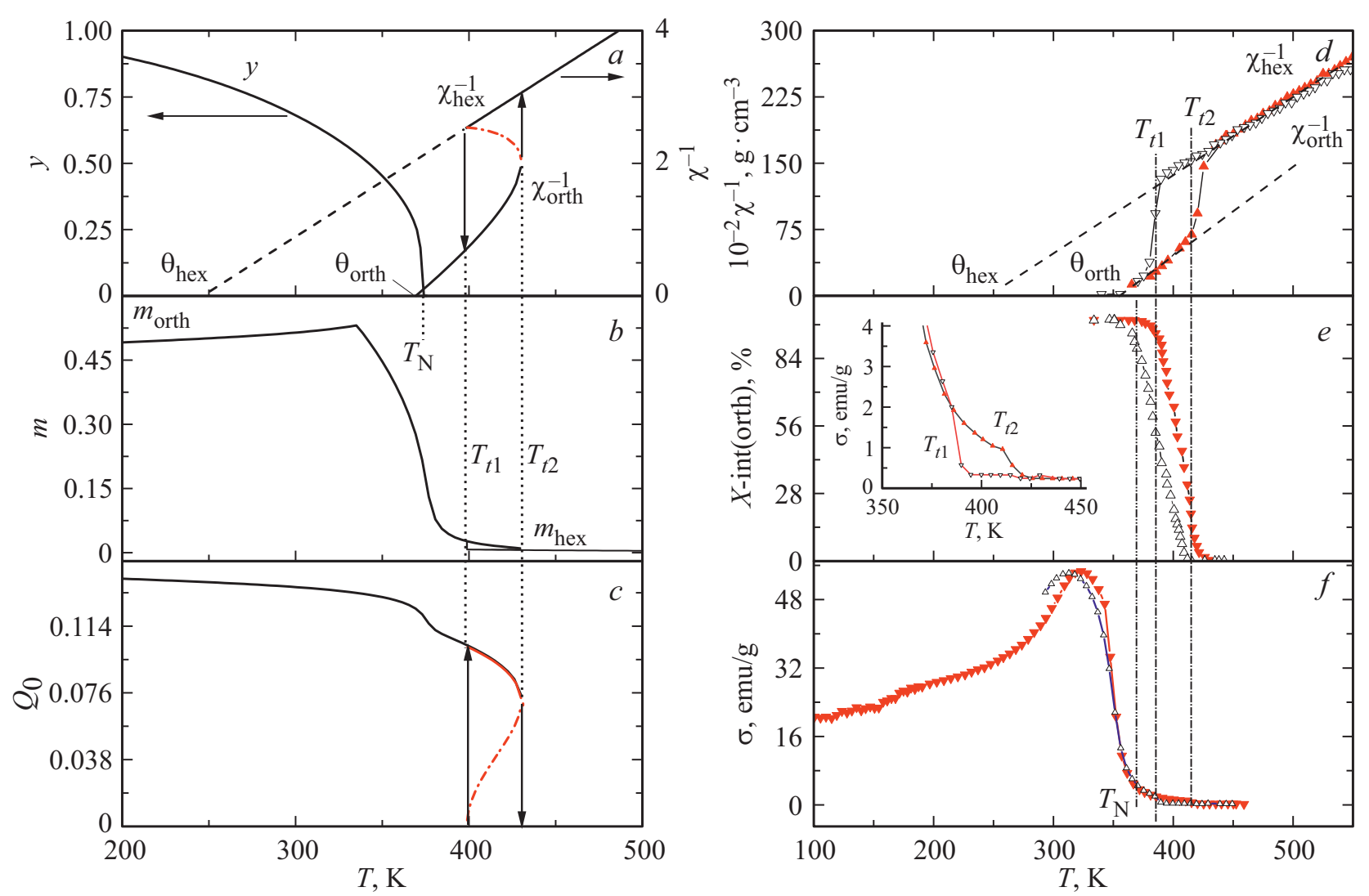

Рис. 1. Теоретические $(a-c)$ и экспериментальные $(d-f)$ зависимости магнитных и рентгеноструктурных характеристик сплава $\mathrm{Mn}_{0.82} \mathrm{Cr}_{0.18} \mathrm{NiGe}$. Теоретические зависимости в безразмерных единицах получены при $\delta=0.925, H=0(a)$ и $H=8.6 \mathrm{kOe}(b, c)$. Экспериментальные зависимости из $[1,3]$ получены при $H=8.6 \mathrm{kOe}(d, f)$ и $H=0(e)$. На вставке фрагмент рис. $1, f$ в области магнитоструктурного периферийного перехода первого рода $P M($ orth $) \leftrightarrow P M($ hex $)$ при $T=400 \mathrm{~K}$.

где

$$
\begin{gathered}
T_{0}=\frac{2}{3 k_{B}} J_{00} s(s+1) ; \quad r F(\delta)=1+\tilde{z}(4 \delta-1) \\
\tilde{z}=\left|J_{20}\right| / J_{00} ; \quad \lambda_{F}=\lambda_{00}+\tilde{z}(4 \delta-1) \lambda_{20} \\
\lambda_{1 F}=\lambda_{01}+\tilde{z}(4 \delta-1) \lambda_{21} .
\end{gathered}
$$

Согласно экспериментальным данным [3], для парамагнитных температур Кюри $\theta_{\text {orth }}$ и $\theta_{\text {hex }}$ ромбической и гексагональной фаз, которые в ПМП совпадают с соответствующими температурами Кюри $\left(\theta_{\text {orth }}=T_{\text {Corth }}\right.$, $\left.\theta_{\text {hex }}=T_{\text {Chex }}\right), \quad$ должно выполняться неравенство $\theta_{\text {hex }} \ll \theta_{\text {orth }}$. Характерные температуры $T_{\text {North }}, T_{\text {Chex }}$, $T_{\text {Corth }}$, согласно экспериментальным данным должны удовлетворять неравенству $T_{t 1}>T_{\text {North }} \geq \theta_{\text {orth }}$. При этом значения этих величин определяются из уравнений (6)

$$
\begin{aligned}
& T_{\text {Corth }} \equiv T 1=\frac{2}{3 k_{B}}\left\{J_{0}\left[Q_{0}(T 1), e_{1}(T 1)\right]\right. \\
& \left.+\left[2(\delta)^{2}+1\right]\left|J_{2}\left[Q_{0}(T 1), e_{1}(T 1)\right]\right|\right\} s(s+1), \delta=1, \\
& T_{\text {Chex }} \equiv T 2=\frac{2}{3 k_{B}}\left\{J_{0}\left[Q_{0}=0, e_{1}(T 2)\right]\right. \\
& \left.+[4(\delta)-1]\left|J_{2}\left[Q_{0}=0, e_{1}(T 2)\right]\right|\right\} s(s+1), \delta=1, \quad(6 \mathrm{~b})
\end{aligned}
$$

$$
\begin{aligned}
& T_{\text {North }} \equiv T 3=\frac{2}{3 k_{B}}\left\{J_{0}\left(Q_{0}(T 3), e_{1}(T 3)\right)\right. \\
& \left.+\left(2(\delta)^{2}+1\right)\left|J_{2}\left(Q_{0}(T 3), e_{1}(T 3)\right)\right|\right\} s(s+1), \delta=1 .
\end{aligned}
$$

Здесь равновесные величины $Q_{0}(T), e_{1}(T, P), e_{2}(T, P)$ определяются из уравнений состояния $\partial \Omega / \partial \vartheta=0$, $\partial \Omega / \partial Q_{0}=0, \partial \Omega / \partial e_{1}=0, \partial \Omega / \partial e_{2}=0, \partial \Omega / \partial \sigma=0$ при $y=0$; для моделирования магнитоструктурного поведения сплавов системы $\mathrm{Mn}_{1-x} \mathrm{Cr}_{x} \mathrm{NiGe}$ следует иметь в виду, что величина $\delta=\cos \Psi$, как важнейшая характеристика гелимагнитной структуры должна возрастать по мере увеличения $x$ от 0.661 до 1 (вблизи $T_{\mathrm{N}}$ ) [4].

Теоретические зависимости $\chi^{-1}\left[T, Q_{0}(T)\right], m(T), y(T)$, $Q_{0}(T)$, рассчитанные при $\delta=0.925, s=2$ и $\delta=1, s=1$ (рис. $1, a-c$, рис. $2, a-c$ ) позволяют объяснить магнитоструктурные особенности экспериментальных зависимостей (рис. $1, d-f$, рис. $2, d-f$ ) обратной восприимчивости $\chi^{-1}(T)$ и намагниченности, впервые приведенных в $[1,3]$, для сплавов с $x=0.11$ и $x=0.18$.

Расчетные зависимости, моделирующие свойства образцов при $x=0.11$ и $x=0.18$, получены с использованием параметров, определенных из нейтронографических и рентгеновских данных при атмосферном давле- 

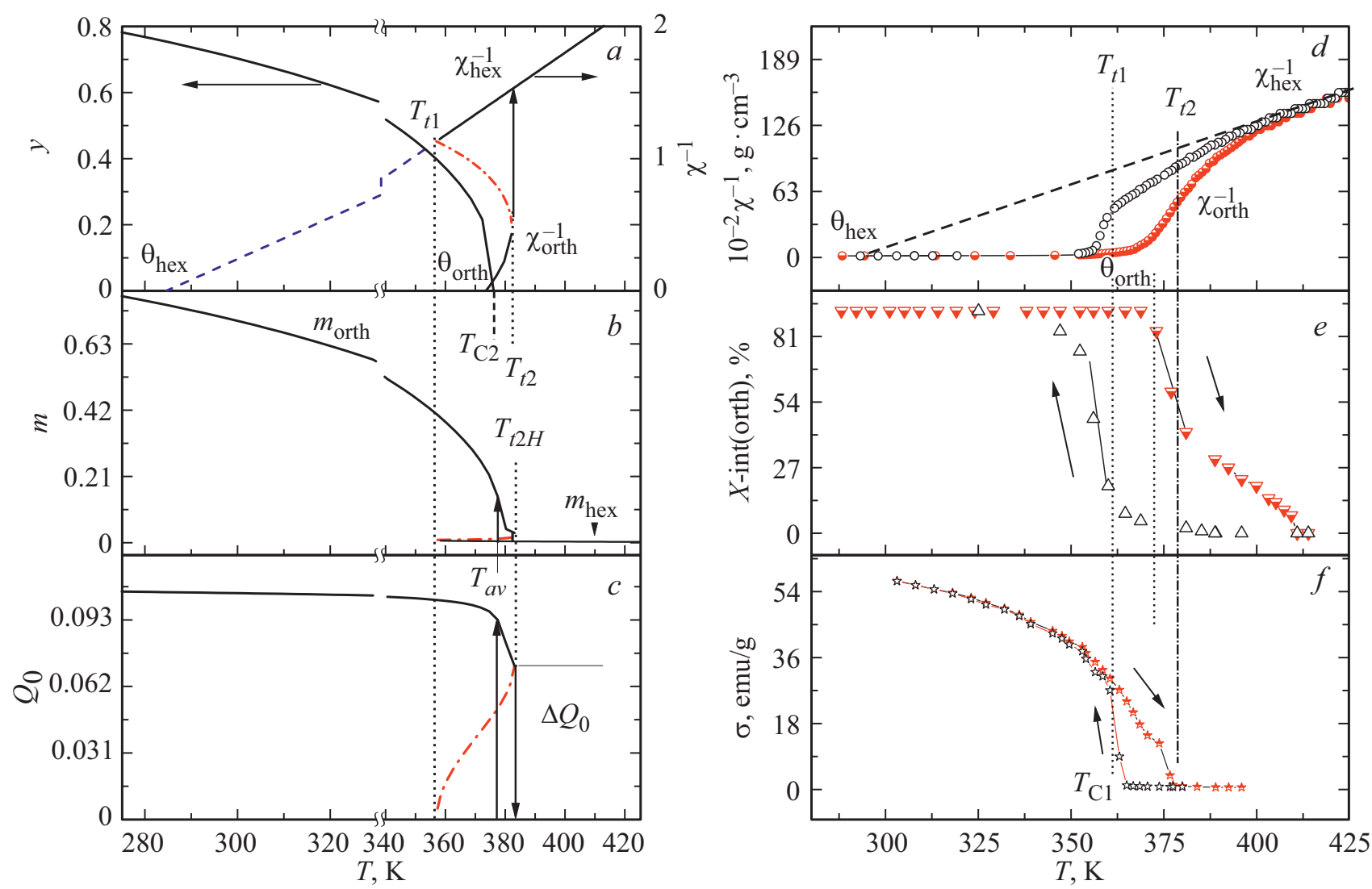

Рис. 2. Теоретические $(a-c)$ и экспериментальные $(d-f)$ зависимости магнитных и рентгеноструктурных характеристик сплава $\mathrm{Mn}_{0.82} \mathrm{Cr}_{0.18} \mathrm{NiGe}$. Теоретические зависимости в безразмерных единицах получены при $\delta=1, H=0(a)$ и $H=9.7 \mathrm{kOe}(b, c)$. Экспериментальные зависимости из [1,3] получены при $H=8.6 \mathrm{kOe}(d), H=0(e)$ и $H=9.7 \mathrm{kOe}(f)$.

нии, а также из магнитных измерений под давлением и термодинамических свойств исследуемой и родственных магнитоструктурных систем.

Согласно рис. $1, c$, рис. 2,c, характерной особенностью безразмерных зависимостей $Q_{T}$, описывающих парамагнитные структурные переходы $h e x\left(P 6_{3} / m m c\right) \rightarrow \operatorname{orth}(P n m a)$, является наличие температурного гистерезиса $\Delta T_{t}=T_{t 2}-T_{t 1}$, внутри которого зависимости $Q_{0}(T)$, обозначенные штрих-пунктирными линиями, соответствуют метастабильным состояниям, т.е. максимумам термодинамического потенциала, и разделяют стабильные ромбическое $\left.\left(Q_{0}\left(T \leq T_{t 1}\right) \neq 0\right)\right)$ и гексагональное $\left(Q_{0}\left(T \geq T_{t 2}\right)=0\right)$ состояния. Ясно, что температуры скачкообразного расщепления зависимости $\chi^{-1}\left(T, Q_{0}\right)$, на две ветви совпадают с температурами лабильности парамагнитных ромбической $T_{t 2}\left(Q_{0} \neq 0\right)$ и гексагональной $T_{t 1}(Q=0)$ фаз.

Принципиальное различие расчетных зависимостей на рис. 1 и рис. 2 состоит в следующем. Для $\delta=0.925$ при температурах $T \leq T_{\mathrm{N}}(\delta)<T_{t 1}$ появляется отличное от нуля значение параметра магнитного порядка геликоидального состояния $y$. Зависимость $y(T)$ при $H_{0 z}=0$ описывает изоструктурный переход второго рода в гелимагнитную (НM) фазу $(P M($ orth $) \leftrightarrow H M($ orth $))$. Температурная зависимость относительной намагниченности
$m(T)$, которая связана с температурно-зависящим параметром гелимагнитного порядка $y(T)$ соотношением $m(T)=y(T) \cos \vartheta(T)$, имеет характерный пик (рис. $1, b)$ и качественно совпадает с экспериментальными зависимостями $\sigma(T)$, рис. $1, f[3]$. Для $\delta=1$ и соответствующего набора постоянных возникает совершенно иная ситуация. Здесь значения температуры лабильности $T_{t 1}$ гексагонального парамагнитного состояния $P M($ hex $)$ ниже температуры $T_{a v}$, которая соответствует равенству термодинамических потенциалов конкурирующих состояний $P M($ hex $)$ и $F M($ orth $)$ и находится из условия $\Omega\left(Q_{0}=0, \sigma, e_{1}, e_{2}=0, y=0\right)=\Omega\left(Q_{0}, \sigma, e_{1}, e_{2}, y\right)$. Поэтому при понижении температуры переход $P M($ hex $) \rightarrow F M($ orth $)$ из гексагонального парамагнитного в ромбическое ферромагнитное состояние может произойти только при температуре $T_{\mathrm{Cl}}$, которая должна удовлетворять неравенству $T_{t 1} \leq T_{\mathrm{Cl}} \leq T_{a v} \leq T_{\mathrm{C} 2}$. Это означает, что скачкообразное возникновение параметра структурного порядка $Q_{0}$ при $T=T_{\mathrm{Cl}}$ влечет за собой скачкообразное возникновение параметра магнитного порядка $y$ (рис. 2,a) и намагниченности $m$ (рис. 2,b), и таким образом магнитоструктурный переход $P M($ hex $) \rightarrow F M($ orth $)$ при $T=T_{\mathrm{Cl}}$ является переходом первого рода. При этом значение $T_{\mathrm{Cl}}$ зависит от конкретного физико-механического состояния образца, 

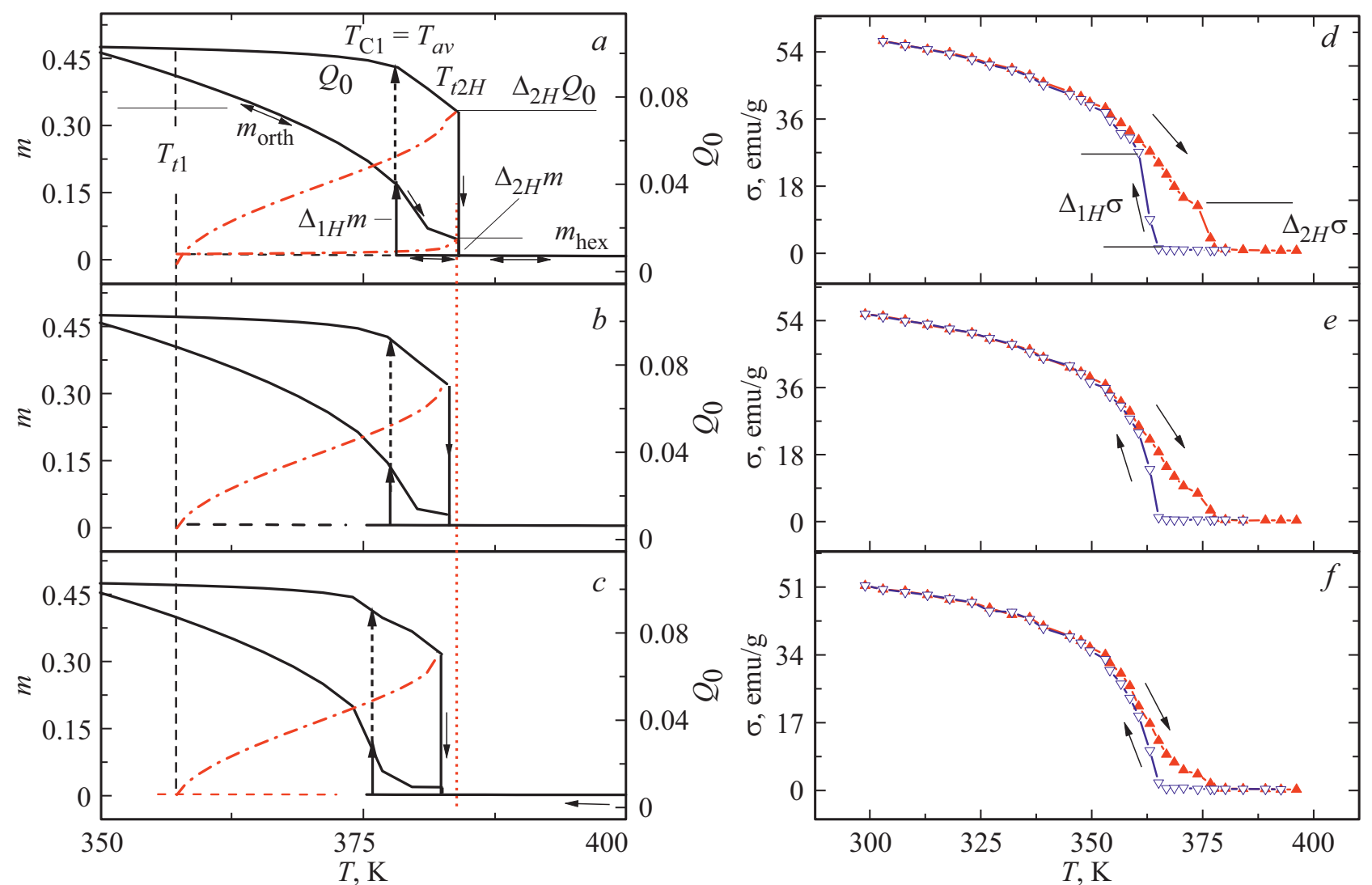

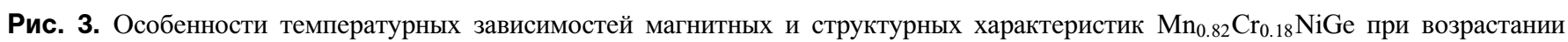
напряженности магнитного поля: $a-c$ - теоретические безразмерные зависимости $m(T) ; d-f-$ экспериментальные зависимости $\sigma(T) ; a, d-H=9.7 \mathrm{kOe} ; b, e-H=6.3 \mathrm{kOe} ; c, f-H=9.7 \mathrm{kOe} ;$ скачок намагниченности $\Delta_{2 H} m$ периферийного участка зависимости $m(T)$ синхронизируется со скачком параметра структурного порядка $\Delta_{2 H} Q_{0}$ при температуре лабильности $T_{t 2 H}$.

которое обусловливает кинетику процесса реализацию перехода (дислокационная структура, степень блокировки зародышеобразования, размытость перехода и иное). На рис. 2, $a-c$ предполагается, что $T_{\mathrm{Cl}}=T_{a v}$. Поэтому понижение температуры ниже $T_{\mathrm{Cl}}=T_{a v}$ не приводит к изменению магнитоструктурного состояния. Обратное повышение температуры при отсутствии магнитного поля приводит к плавному изоструктурному исчезновению магнитного порядка, реализующемуся как изоструктурный переход второго рода $F M($ orth $) \rightarrow P M($ orth $)$, поскольку $T_{\mathrm{C} 2}<T_{t 2}$. В присутствии магнитного поля подмагниченное полем ромбическое состояние не полностью исчезает при $T=T_{\mathrm{C} 2}$ (рис. $\left.2, b\right)$ и затягивается к температурам $T_{t 2 H}$, превосходящим температуры спонтанного исчезновения ромбического состояния $T_{t 2 H} \geq T_{t 2}$. Поэтому скачкообразное исчезновение параметра структурного порядка $\Delta_{2 H} Q_{0}=Q_{0}\left(T_{t 2 H}\right)$ (рис. $2, c)$ влечет за собой скачкообразное уменьшение намагниченности на периферийном участке зависимости $m_{\text {orth }}(T)$ при $T_{t 2 H}$ до ее значения в гексагональном состоянии $m_{h e x}$. При этом скачок намагниченности $\Delta_{2 H} m=m_{\text {orth }}-m_{\text {hex }}$ и скачок параметра структурного порядка $\Delta_{2 H} Q_{0}=Q_{0}\left(T_{t 2 H}\right)$ при $T=T_{t 2 H}$ увеличиваются при возрастании магнитного поля (рис. 3). Поскольку
$T_{t 2 H}>T_{t 2}, \quad$ то можно говорить об увеличении температурного гистерезиса инверсионного перехода $F M($ orth $) \rightarrow P M($ hex $)$ при возрастании напряженности магнитного поля. Более подробно это иллюстрируется экспериментальной зависимостью $\sigma(T)$ и теоретическими зависимостями $m(T), Q_{0}(T)$, приведенными на рис. 3 . Таким образом, аномальное затягивание остаточной намагниченности $m(T)$ (рис. 2,b) в область высоких температур и ее скачкообразное снижение на периферийном участке связано со смещением температуры лабильности ромбической фазы (рис. $2, c$ ) в магнитном поле от $T_{t 2}$ при $H=0$ до $T_{t 2 H}$ при $H=8.6 \mathrm{kOe}$. В работе [3] эти высокотемпературные скачки намагниченности на периферийных участках экспериментальных (теоретических) зависимостей $\sigma(T),(m(T))$, которые являются причиной аномалий обратной восприимчивости, определяются как „периферийные“ магнитоструктурные переходы первого рода. В отсутствие магнитного поля „периферийные“ магнитоструктурные переходы первого рода трансформируются в структурные переходы первого рода $P M(P n m a) \rightarrow P M\left(P 6_{3} / m m c\right)$, которые сопровождаются только скачкообразными изменениями параметра структурного порядка $Q_{0}(T)$. 

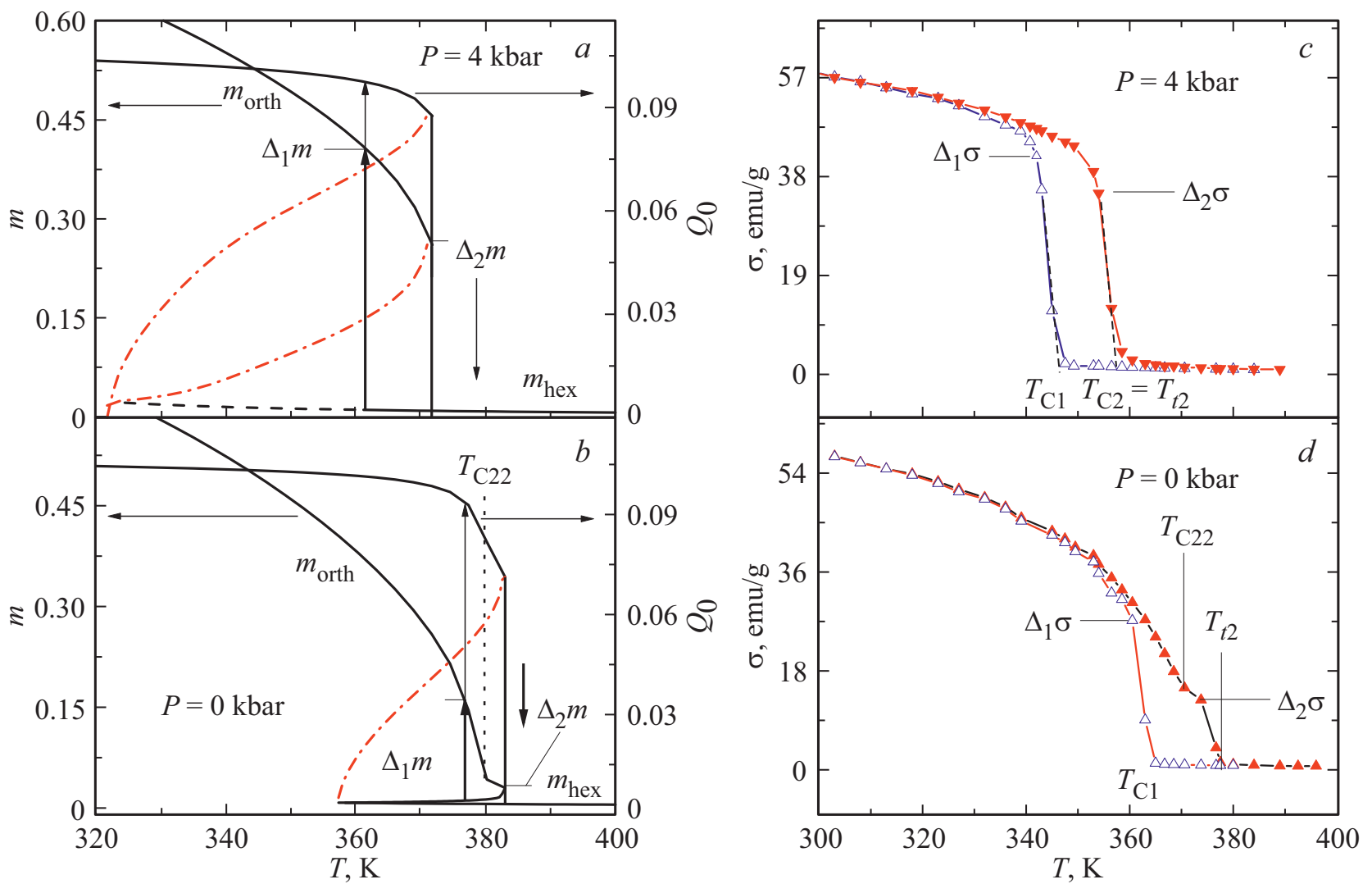

Рис. 4. Трансформация реверсивного перехода под действием давления в поле $H=9.7 \mathrm{kOe:} a, b-$ теоретическая зависимость (безразмерные единицы); $c, d-$ эксперимент. $T_{\mathrm{C} 22}$ - температура максимального спада намагниченности образца в области ромбической фазы при нагревании, которая при $H=0$ соответствует температуре Кюри $T_{\mathrm{C} 2}$ (рис. $2, a$ ) изоструктурного перехода второго рода $F M($ orth $) \rightarrow P M($ orth $)$.

В сплавах с $x<0.18$, когда температурные границы устойчивости структурного и магнитного параметров порядка значительно разнесены, стабилизация структурного параметра порядка приводит только к смещению магнитного упорядочения из области более низких в область более высоких температур $\left(\theta_{\text {hex }}<\theta_{\text {orth }}<T_{N}<T_{t 1}\right)$ и поэтому возникновение и исчезновение параметра магнитного порядка происходит при одной и той же температуре и может быть охарактеризовано как изоструктурный переход второго рода $H M($ orth $) \leftrightarrow P M($ orth $)$ (рис. 1,a). Возникновение реверсивного перехода первого рода в этом случае $(x=0.11)$ возможно при сближении характеристических температур магнитного и структурного переходов. Такое сближение может происходить при воздействии гидростатического давления, что приводит к трансформации изоструктурного перехода второго рода $H M($ orth $) \leftrightarrow P M($ orth $)$ к реверсивному магнитоструктурному переходу 1-го рода $P M($ hex $) \rightarrow H M($ orth $)$ [3]. Для образцов с $x=0.18$ барическое сближение характеристических температур магнитного и структурного переходов, напротив, приводит к исчезновению реверсивных переходов и возникновению полно- ценных магнитоструктурных переходов первого рода $F M($ orth $) \leftrightarrow P M($ hex $)$.

Теоретически это было предсказано в [1] при рассмотрении обобщенной $P-T$-диаграммы системы с сильным взаимодействием параметров магнитного и структурного порядков. В настоящей работе трансформация реверсивного магнитоструктурного перехода первого рода в полноценный магнитоструктурный переход первого рода при увеличении давления иллюстрируется теоретическими зависимостями $m(T), Q_{0}(T)$ при $P=0$ и $P=4 \mathrm{kbar}$ (рис. $4, a, b$ ) при значениях параметров, соответствующих $\delta=1$. Экспериментальные зависимости намагниченности от температуры при этих давлениях для $\mathrm{Mn}_{0.82} \mathrm{Cr}_{0.18} \mathrm{NiGe}$ (рис. $4, c, d$ ) полностью подтверждают теорию. Как видно из графиков на рис. 4, трансформация реверсивных переходов связана с возрастанием скачков намагниченности от $\Delta_{1} m\left(T_{\mathrm{Cl}}\right)<1, \Delta_{2} m\left(T_{t 2}\right) \ll 1$ при атмосферном давлении $(P=0$, рис. $4, b)$ до $\Delta_{2} m\left(T_{t 2}\right) \leq \Delta_{1} m\left(T_{t 1}\right) \leq 1$ при $P=4$ kbar, pис. $4, b$. Сближение и совмещение характерных температур под действием давления при магнитоструктурных переходах отслеживается на экспериментальной и теоретической $\mathrm{P}-\mathrm{T}$-диаграммах исследуемого сплава (рис. 5). 

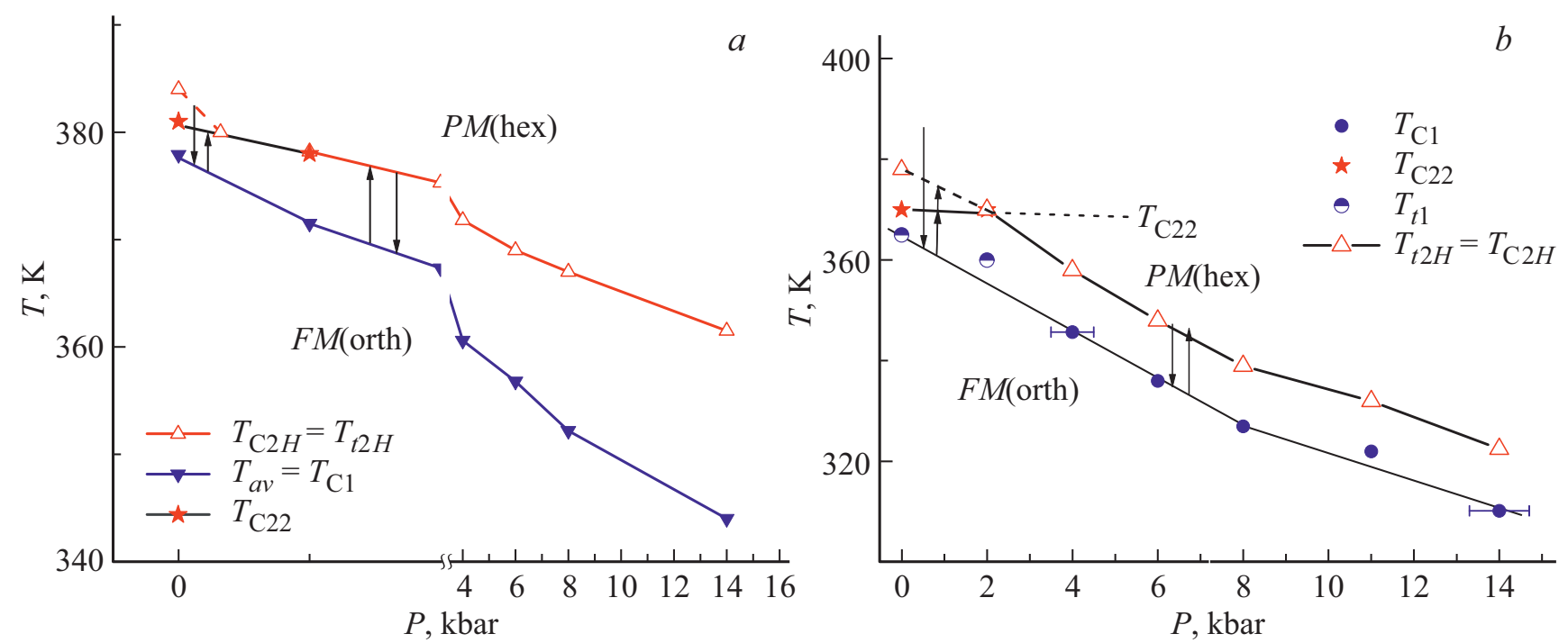

Рис. 5. Теоретическая (a) и экспериментальная $(b) P-T$-диаграммы сплава $\mathrm{Mn}_{0.82} \mathrm{Cr}_{0.18} \mathrm{NiGe}$ в поле $H=9.7 \mathrm{kOe}$.

\section{4. Магнитокалорические особенности реверсивных переходов}

Выражение для энтропии системы $S \equiv S\left[Q_{0}(T, H), y(t, H), T\right]$ можно получить из определения $S=-\partial \Omega / \partial T$ и привести к виду

$$
\begin{aligned}
S[ & \left.Q_{0}(T, H), y(T, H), T\right] \\
= & N k_{B} \ln \left\{z\left[X\left(Q_{0}(T, H), y(T, H), T\right)\right]\right\} \\
& -N k_{B} y(T, H) X\left(Q_{0}(T, H), y(t, H), T\right) \\
& +\alpha k_{0} e_{1}\left(Q_{0}(T), y(T, H), T\right)+\frac{1}{2} N_{0} k_{B} \ln \left(Q_{0}(T, H), T\right) .
\end{aligned}
$$

В (7) первые два слагаемых соответствуют энтропии магнитоупорядоченной системы спинов в ромбической $\left(Q_{0} \neq 0\right)$ или гексагональной $\left(Q_{0}=0\right)$ кристаллических решетках при заданной температуре. Третье слагаемое - энтропия объемно-деформированного магнитоупорядоченного кристалла в ромбическом (гексагональном) состояниях. Последнее слагаемое описывает понижение энтропии кристалла, обусловленное изменением величины параметра структурного порядка $Q_{0}$. Зависимости $S\left[Q_{0}(T, H), y(T, H), T\right]$ от температуры в магнитном поле $(H=9.7 \mathrm{kOe})$ и без поля $(H=0)$ представлены на рис. 6.

Можно ожидать, что эта зависимость будет проявлять реверсивные свойства и существенные ее изменения зависят от последовательности изменения температуры (повышения, понижения температуры). Это наиболее отчетливо проявляется на изотермической зависимости $\Delta S(T, H)=S\left[Q_{0}(T, H), y(T, H), T\right]$ - $\left[Q_{0}(T, 0), y(T, 0), T\right]$ (рис. 7), которая используется для оценки магнитокалорического эффекта в практи-

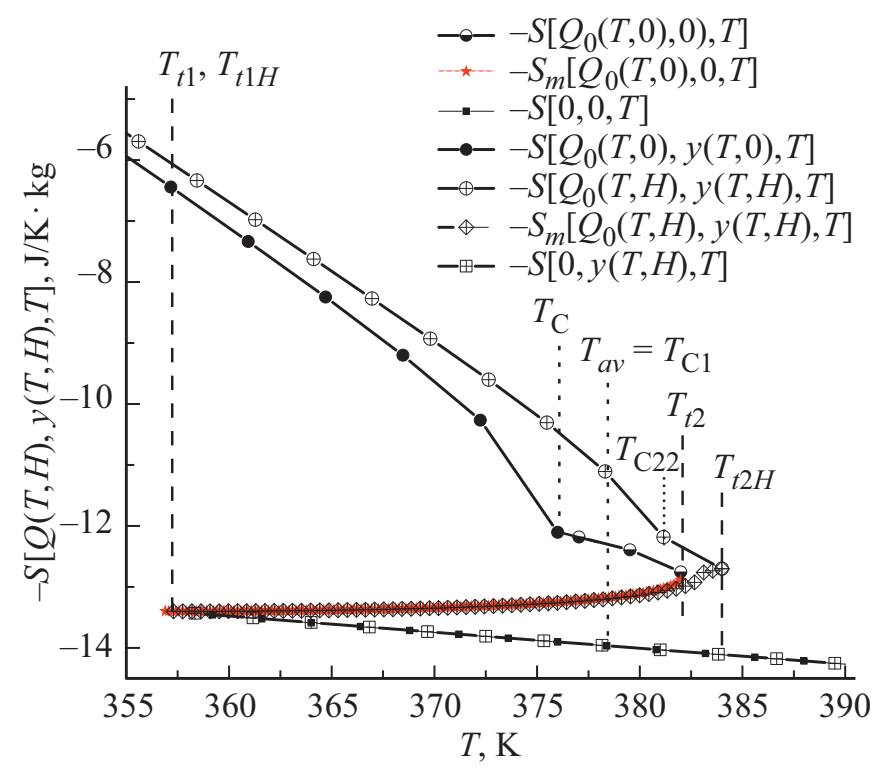

Рис. 6. Теоретическая температурная зависимость энтропии системы в процессе реализации инверсионного магнитоструктурного перехода первого рода в магнитном поле $H=9.7 \mathrm{kOе}$ и без поля. Вертикальные штриховые линии отмечают температуры лабильности ромбической и гексагональной фаз в поле $H=9.7 \mathrm{kOe}$ и без поля; зависимости с индексом $m\left(S_{m}\right)$ соответствуют максимумам термодинамического потенциала; $T_{C 22}, T_{\mathrm{C}}-$ температуры изоструктурных переходов $F M($ orth $) \rightarrow P M($ orth $)$ в поле и без поля при нагревании.

ческих приложениях. На рис. 7 сопоставлены теоретическая и экспериментальная (полученная методом Максвелла [10]) зависимости $\Delta S(T, H)$.

При понижении температуры от состояния $P M($ hex $)$ в области $T_{t 1} \leq T_{\mathrm{Cl}}<T$ величины $\Delta S(T, H)$ на рис. $7, b$ определяются выражением. При $T=T_{\mathrm{Cl}}$ величина 

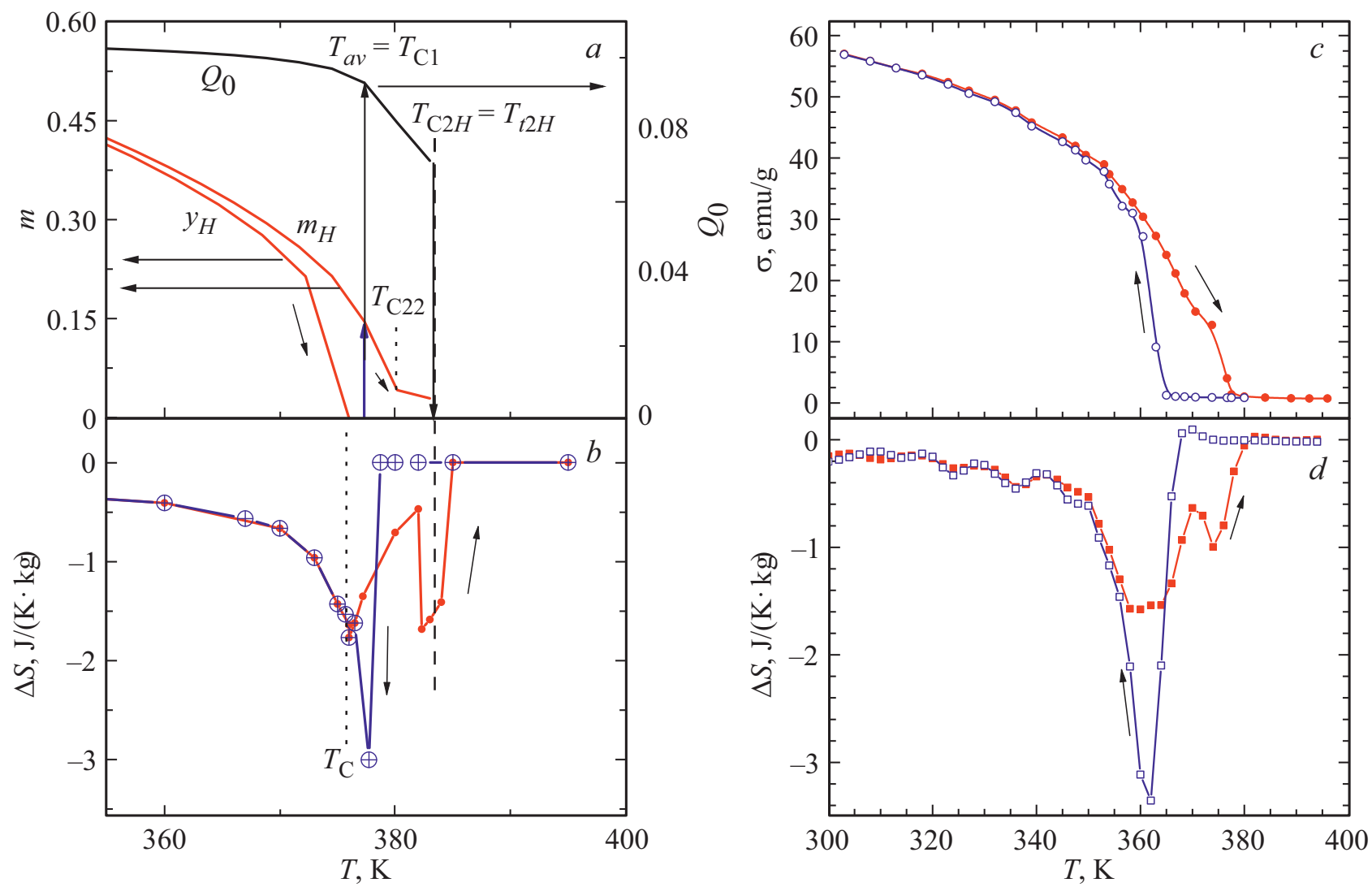

Рис. 7. Магнитоструктурные характеристики $\mathrm{Mn}_{0.82} \mathrm{Cr}_{0.18} \mathrm{NiGe}(a, c)$, совмещенные с теоретической $(b)$ и экспериментальной $(d)$ температурными зависимостями изменения магнитной энтропии $\Delta S(T)=S(T, H=9.7 \mathrm{kOe})-S(T, H=0) ; a-$ теоретические температурные зависимости безразмерной намагниченности $m_{H}=y_{H} \cos (\vartheta(H))$, параметра структурного порядка $Q_{0}$ в поле $H=9.7 \mathrm{kOe}$ и параметра магнитного порядка $y_{H}$ при $H=0 ; c-$ экспериментальная температурная зависимость намагниченности $\sigma$ в поле $H=9.7 \mathrm{kOe}$. Экспериментальные зависимости $\Delta S(T)$ получены по данным магнитных измерений с использованием соотношений Максвелла.

$\Delta S\left(T_{\mathrm{Cl}}, H\right)=S\left[Q_{0}, y\left(T_{\mathrm{Cl}}, H\right), T_{\mathrm{Cl}}\right]-S\left[0, y\left(T_{\mathrm{Cl}}, 0\right), T_{\mathrm{Cl}}\right]$ определяет резкий пик зависимости $\Delta S(T, H)$. Этот пик соответствует магнитоструктурному переходу первого рода $P M($ hex $) \rightarrow F M($ orth $)$. Дальнейшее понижение температуры формирует плавную спадающую зависимость $\Delta S(T, H)$, определяемую выражением: $\Delta S(T, H)=$ $=S\left[Q_{0}, y(T, H), T\right]-S[0, y(T, 0), T]$.

Таким образом, понижение температуры характеризуется однопиковой структурой зависимости $\Delta S(T, H)$. Напротив, при повышении температуры можно выделить два заметных пика. Высокотемпературный пик, который определяется разностью $\Delta S\left(T_{\mathrm{C} 2}, \Delta H\right)=$ $=S\left[Q_{0}\left(T_{\mathrm{C} 2}, H\right), y\left(T_{\mathrm{C} 2}, H\right), T_{\mathrm{C} 2}\right]-S\left[Q_{0}\left(T_{\mathrm{C} 2}, 0\right), 0, T_{\mathrm{C} 2}\right]$ coответствует изоструктурному переходу второго рода $F M($ orth $) \leftrightarrow P M($ orth $)$. Низкотемпературный пик определяется разностью $S\left[Q_{0}(T, H), y(T, H), T_{t}\right]-S\left[0,0, T_{t}\right]$ в окрестности $T_{t 2 H}$ и соответствует парамагнитному структурному переходу первого рода $P M($ orth $) \rightarrow P M($ hex $)$. Как видно из сопоставления экспериментальных и теоретических графиков на рис. 7 , обе зависимости $\Delta S(T, \Delta H)$ находятся в хорошем качественном соответствии.

\section{5. Заключение}

Теоретический анализ, проведенный в настоящей работе, позволил объяснить магнитные и магнитокалорические особенности магнитноструктурных фазовых переходов, которые реализуются в системах с кристаллоструктурной неустойчивостью.

1. При определенных соотношениях между обменными и структурными параметрами, ответственными за возникновение низкосимметричных магнитного и структурного параметров порядка, возможна реализация реверсивных переходов первого рода. При реверсивных переходах охлаждение образца приводит к магнитоструктурному переходу первого рода $P M($ hex $) \rightarrow F M($ orth $)$; при нагревании образца реализуются два фазовых перехода. Магнитное разупорядочение описывается изоструктурным переходом второго рода $F M($ orth $) \leftrightarrow P M($ orth $)$. Затем при более высоких температурах реализуется парамагнитный структурный переход $P M($ orth $) \rightarrow P M($ hex $)$. В магнитном поле этот переход реализуется как периферийный магнитоструктурный переход первого рода с небольшим конечным скачком 
намагниченности. Периферийный переход ответственен за аномальное поведение обратной магнитной восприимчивости.

2. Особенностью периферийных магнитоструктурных переходов первого рода является возрастание скачка намагниченности при возрастании напряженности магнитного поля.

3. Последовательность скачкообразных изменений намагниченности при реверсивных переходах определяет характерную реверсивную последовательность пиков изотермической зависимости $\Delta S(T, H)$.

4. Воздействие давления на реверсивные переходы приводит к трансформации их в полноценные магнитоструктурные переходы первого рода $P M($ hex $) \leftrightarrow F M($ orth $)$.

\section{Финансирование работы}

Работа выполнена при поддержке гранта Российского научного фонда (проект № 20-19-00745).

\section{Конфликт интересов}

Авторы заявляют, что у них нет конфликта интересов.

\section{Приложение}

Гамильтониан спиновой подсистемы в присутствии внешнего магнитного поля $\mathbf{H}_{0}$ можно записать в виде

$$
\hat{\mathbf{H}}_{s}=-\sum_{n k, n^{\prime} k^{\prime}} J_{n n^{\prime}}^{k k^{\prime}} \hat{\mathbf{s}}_{n}^{k} \hat{\mathbf{s}}_{n^{\prime}}^{k^{\prime}}-2 \mu_{0} \mathbf{H}_{0} \sum_{n i} \hat{\mathbf{s}}_{n}^{k},
$$

где $\hat{\mathbf{s}}_{n}^{k}$ - оператор спина атома $k$ в элементарной гексагональной ячейке $n ; \mathbf{J}_{n n^{\prime}}^{k k^{\prime}} \equiv J\left(\left|\Delta \mathbf{R}_{n n^{\prime}}^{k k^{\prime}}\right|\right)-$ соответствующие интегралы обменного взаимодействия между магнитоактивными атомам на расстояниях $\left|\Delta \mathbf{R}_{n n^{\prime}}^{k k^{\prime}}\right|$; $\sum_{n} N_{0}, \sum_{n, k}=N=2 N_{0} x$ - число элементарных ячеек и число магнитоактивных атомов $(\mathrm{Mn})$ в единице объема, $\mathbf{H}_{0}=\left(0,0, H_{0}\right)$ - вектор внешнего однородного магнитного поля, $\mu_{0}$ - магнетон Бора.

Для простого гелимагнетика (НМ) с волновым вектором $\mathbf{k}=\left(0,0, k_{\mathrm{a}}\right)$ угол $\Psi_{n n^{\prime}}^{k k^{\prime}}$ между направлениями средних значений спинов атомов, находящихся на расстояниях $\left|\Delta \mathbf{R}_{n n^{\prime}}^{k k^{\prime}}\right|$, определяется выражением $\Psi_{n n^{\prime}}^{k k^{\prime}}=\mathbf{k}\left(\mathbf{R}_{n^{\prime}}^{k^{\prime}}-\mathbf{R}_{n}^{k}\right) \equiv \mathbf{k} \Delta \mathbf{R}_{n n^{\prime}}^{k k^{\prime}}=k_{a} c_{n n}^{k k^{\prime}}\left(c_{n n}^{k k^{\prime}}-\right.$ pacстояние между атомамив $k n, k^{\prime} n^{\prime}$ вдоль направления волнового вектор $\mathbf{k}$. Для описания НМ-упорядочения в приближении среднего поля используем гамильтониан (П2), в котором направление пространственно-неоднородного среднего поля $\mathbf{h}_{n}^{k}=h \mathbf{U}_{n}^{k}$ для атома в позиции $\mathbf{R}_{n}^{k}$ определяется единичным вектором $\left.\mathbf{U}_{n}^{k}=\left(\cos \left(\mathbf{k} \mathbf{R}_{n}^{k}\right)\right) \sin (\vartheta) \sin \left(\mathbf{k} \mathbf{R}_{n}^{k}\right) \sin (\vartheta), \cos (\vartheta)\right)$ и совпадает с направлением локальной оси квантования. Предполагается, что при $H_{0}=0$ локальная ось квантования находится в базовой плоскости, перепендикулярной $\mathbf{k}$, $(\vartheta=\pi / 2)$. Модельный гамильтониан $\hat{\mathbf{H}}_{h s}$ и модельная свободная энергия $\Omega_{h} \equiv \Omega(h)$ определяются выражениями

$$
\begin{gathered}
\hat{\mathbf{H}}_{h s}=-\sum_{n k} h \mathbf{U}_{n}^{k} \hat{\mathbf{s}}_{n}^{k}=-\sum_{n k} h \hat{m}_{n}^{k}, \\
\Omega_{h}=\left\langle\hat{\mathbf{H}}_{s}-\hat{\mathbf{H}}_{h s}\right\rangle_{h}-N k_{B} T \ln [z(X)],
\end{gathered}
$$

$\hat{m}_{n}^{k}=\mathbf{U}_{n}^{k} \hat{\mathbf{s}}_{n}^{k}-$ оператор проекции спина на ось квантования,

$$
\begin{aligned}
& \left\langle\hat{\mathbf{H}}_{s}-\hat{\mathbf{H}}_{h s}\right\rangle_{h}=-\sum_{n, k}\left[\left[\sum_{n^{\prime}, k^{\prime}} J\left(\Delta \mathbf{R}_{n n^{\prime}}^{k k^{\prime}}\right)\left\langle\hat{\mathbf{s}}_{n}^{k}\right\rangle_{h}\left\langle\hat{\mathbf{s}}_{n^{\prime}}^{k^{\prime}}\right\rangle_{h}\right]\right. \\
& \left.+2 \mu_{0} \mathbf{H}_{0}\left\langle\hat{\mathbf{s}}_{n}^{k}\right\rangle-\langle\hat{m}\rangle_{h} h\right]=-\sum_{n, k}\left[\left[\langle\hat{m}\rangle_{h}^{2} \sum_{n^{\prime}, k^{\prime}} J\left(\Delta \mathbf{R}_{n n^{\prime}}^{k k^{\prime}}\right) \mathbf{U}_{n}^{k} \mathbf{U}_{n^{\prime}}^{k^{\prime}}\right]\right. \\
& \left.+2 \mu_{0} \mathbf{H}_{0} \mathbf{U}_{n}^{k}\langle\hat{m}\rangle_{h}-\langle\hat{m}\rangle_{h} h\right]=-N\langle\hat{m}\rangle_{h}^{2}\left[J(k) \sin ^{2}(\vartheta)\right. \\
& \left.+J(0) \cos ^{2}(\vartheta)\right]-N 2 \mu_{0} H_{0} \cos (\vartheta)\langle\hat{m}\rangle_{h}+N\langle\hat{m}\rangle_{h} h .
\end{aligned}
$$

В (П4а) учтено, что $\mathbf{H}_{0} \mathbf{U}_{n}^{k}=H_{0} \cos (\vartheta)$ и вклад в диагональные собственные значения $\hat{\mathbf{s}}_{n}^{k}$ будут давать лишь компоненты $\hat{m}_{n}^{k}$, направленные вдоль среднего поля, поэтому связь между средним значением спина $\left\langle\hat{\mathbf{s}}_{n}^{k}\right\rangle_{h}$ и $\left\langle\hat{m}_{n}^{k}\right\rangle_{h}$ в приближении среднего поля определяется выражением

$$
\left\langle\hat{\mathbf{s}}_{n}^{k}\right\rangle_{h}=\mathbf{U}_{n}^{k}\left\langle\hat{m}_{n}^{k}\right\rangle_{h}=\mathbf{U}_{n}^{k}\langle\hat{m}\rangle_{h}=\mathbf{U}_{n}^{k} y s .
$$

Усреднение в рамках ПСП проводится по схеме

$$
\begin{gathered}
y s=\langle\hat{m}\rangle_{h}=\operatorname{Sp} \hat{m} e^{\beta h \hat{m}} / z(x)=\sum_{m=-s}^{s} m e^{\beta h m} / z(X) \\
\equiv \beta^{-1} \partial \ln z(X) / \partial h=s B_{s}(X) \\
=s\left[\left(\frac{1}{2 s+1}\right) \operatorname{coth} \frac{1}{2 s+1} X-\left(\frac{1}{2 s}\right) \operatorname{coth} \frac{1}{2 s} X\right], \\
z(X)=\operatorname{Sp} e^{\beta h \hat{m}_{n}^{k}}=\sum_{m=-s}^{s} e^{\beta h m_{n}^{k}} \\
=\operatorname{sh}\left[\left(1+(2 s)^{-1}\right) X\right] / \operatorname{sh}\left[(2 s)^{-1} X\right], \\
X=\beta s h .
\end{gathered}
$$

В приближении ближайших и следующих за ближайшими атомами Mn в плоскостях перпендикулярных волновому вектору $\mathbf{k} J\left(k_{a}\right)$ имеет вид

$$
\begin{array}{rr}
J\left(k_{a}\right)=\sum_{\Delta R} J(|\Delta \mathbf{R}|) \cos (\mathbf{k} \Delta \mathbf{R}) \approx J_{0}+J_{1} \cos (\Psi)+J_{2} \cos (2 \Psi), \\
J(0) \equiv J\left(k_{a}=0\right) \equiv J(\Psi=0)=J_{0}+J_{1}+J_{2} . & (\Pi 6 \mathrm{~d})
\end{array}
$$


С учетом (П4-П6) $\Omega_{h}$ приобретает вид

$$
\begin{aligned}
\Omega_{h}= & -N s^{2} y^{2}\left[J\left(k_{a}\right) \sin ^{2}(\vartheta)+J(0) \cos ^{2}(\vartheta)\right] \\
& -N s y 2 \mu_{0} H_{0} \cos (\vartheta)+N s y h-N k_{B} T \ln [z(X)] .
\end{aligned}
$$

Выражение для модуля среднего поля $h$ определяется из условия $d \Omega_{h} / d h=\partial \Omega_{h} / \partial h+\left(\partial \Omega_{h} / \partial y\right) d y / d h=0$, которое с учетом (П5) приводится к виду

$$
\begin{aligned}
(d y / d h)\left\{-2 s y\left[J\left(k_{a}\right)\right.\right. & \left.\sin ^{2}(\vartheta)+J(0) \cos ^{2}(\vartheta)\right] \\
& \left.-2 \mu_{0} H_{0} \cos (\vartheta) s+h\right\}=0 .
\end{aligned}
$$

Отсюда

$$
h=2 s y\left[J\left(k_{a}\right) \sin ^{2}(\vartheta)+J(0) \cos ^{2}(\vartheta)\right]+2 \mu_{0} H_{0} \cos (\vartheta) s .
$$

После подстановки в (П7) выражения для $h \Omega_{h} \equiv \Omega(h)$ приобретает вид $\Omega_{2}(y)$ в (3c).

\section{Список литературы}

[1] В.И. Вальков, В.И. Каменев, В.И. Митюк, И.Ф. Грибанов, А.В. Головчан, Т.Ю. Деликатная. ФТТ 59, 266 (2017).

[2] В.И. Вальков, И.Ф. Грибанов, Б.М. Тодрис, А.В. Головчан, В.И. Митюк. ФТТ 60, 1113 (2018).

[3] В.И. Вальков, А.В. Головчан, В.В. Коледов, Б.М. Тодрис, В.И. Митюк. ФТТ 62, 710 (2020).

[4] B. Penc, A. Hoser, S. Baran, A. Szytuła. Phase Transit. 91, 118 (2018).

[5] J. Łažewski, P. Piekarz, K. Parlinski. Phys. Rev. B 83, 054108 (2011).

[6] Р. Блинц, Б. Жекш. Сегнетоэлектрики и антисегнетоэлектрики. Динамика решетки. Мир, М. (1975).

[7] A.I. Liechtenstein, M.I. Katsnelson, V.P. Antropov, V.A. Gubanov. JMMM 67, 65 (1987).

[8] I. Rungger, S. Sanvito. Phys. Rev. B 74, 024429 (2006).

[9] L.M. Sandratskii, E. Sasioglu. Phys. Rev. B 74, 214422 (2006).

Редактор Е.Ю. Флегонтова 